\title{
Classroom Activity: Kepler's Laws of Planetary Motion
}

\author{
By Y. Tsubota
}

Keio Senior High School, 4-1-2 Hiyoshi, Kouhoku-ku, Yokohama 223, JAPAN

\section{Introduction}

This activity was originally developed by a group of teachers in Japan during 1960s under the influence of the American Curriculum-Reform Movement. This was used in Earth Sciences in order to develop the students' cognitive skill. Kepler had been trying to analyze Tycho's observations of Mars, fitting them into the Copernican orbital system. It simply would not work. The problem is with the circular orbit that the Copernican system still used. Mars obviously did not have a circular orbit about the Sun. So Kepler tried a variety of other geometrical shapes, until he finally found the ellipse. This worked for all of the planets as well. Kepler's law of planetary motion is cursorily mentioned in most secondary Earth Science and Physics textbooks as shown in Table 1.

\begin{tabular}{|l|c|l|}
\hline Textbook & Chapter & Chapter Title \\
\hline $\begin{array}{l}\text { SPACESHIP EARTH Earth Science } \\
\text { HoughtonMifflin, 1984 }\end{array}$ & 4 & SolarSystem \\
\hline $\begin{array}{l}\text { Modern Earth Science } \\
\text { Holt, Rinehart and Winston, 1989 }\end{array}$ & 29 & The Solar System \\
\hline $\begin{array}{l}\text { Investigating the Earth } \\
\text { Houghton Mifflin, 1991 }\end{array}$ & 1 & $\begin{array}{l}\text { The Earth in Space } \\
\text { The Solar System }\end{array}$ \\
\hline $\begin{array}{l}\text { Earth Science } \\
\text { Scoot, Foresman }\end{array}$ & 23 & The Solar System \\
\hline $\begin{array}{l}\text { PHYSICS } \\
\text { AddisonWesley, 1991 }\end{array}$ & 6 & Circular Motion and Gravitation \\
\hline $\begin{array}{l}\text { PHYSICAL SCIENCE } \\
\text { Scoot, Foresman }\end{array}$ & 4 & Graviton \\
\hline
\end{tabular}

Table 1. Correlation of this experiment with American science textbooks.

But the objectives of this activity covers Kepler's Law in greater detail. After completing this activity, students will be able to understand Kepler's method to determine an orbit of Mars; understand Kepler's laws of planetary motion; and describe the planetary motion. Students will also learn about the history of science and nature of the scientific belief. This 90 -minutes activity is divided into three parts: drawing the orbit of Mars sixty minutes; analysis - fifteen minutes, and post-lab discussion - fifteen minutes.

\section{Student Activity}

\subsection{Materials For Each Student}

Each student needs following materials: a ruler, a circular protractor, a compass, graph paper, observational data as shown in Table 2. 


\begin{tabular}{|l|l|r|r|r|r|}
\hline Mars & Earth & \multicolumn{1}{l|}{ Date } & \multicolumn{1}{l|}{ Sun } & \multicolumn{1}{l|}{ Mars } & Mars-Sun \\
\hline M1 & E1 & $7 / 28 / 69$ & 124.8 & 244.4 & 119.6 \\
\hline & E1' & $6 / 15 / 71$ & 83.3 & 317.8 & 234.5 \\
\hline M2 & E2 & $9 / 16 / 71$ & 172.5 & 312.3 & 139.8 \\
\hline & E2' & $8 / 3 / 73$ & 130.5 & 25.4 & -105.1 \\
\hline M3 & E3 & $11 / 4 / 73$ & 221.4 & 28.5 & -192.9 \\
\hline & E3' & $9 / 22 / 75$ & 178.4 & 79.4 & -99.0 \\
\hline M4 & E4 & $12 / 24 / 75$ & 271.5 & 78.1 & -193.4 \\
\hline & E4' & $11 / 10 / 77$ & 227.5 & 125.4 & -102.1 \\
\hline M5 & E5 & $2 / 11 / 78$ & 321.9 & 114.7 & -207.2 \\
\hline & E5' & $12 / 30 / 79$ & 277.7 & 163.5 & -114.2 \\
\hline M6 & E6 & $4 / 1 / 80$ & 11.4 & 146.0 & 134.6 \\
\hline & E6' & $2 / 17 / 82$ & 328.8 & 199.0 & -129.8 \\
\hline M7 & E7 & $5 / 21 / 82$ & 59.6 & 180.9 & 121.3 \\
\hline & E7' & $4 / 7 / 84$ & 17.4 & 238.2 & 220.8 \\
\hline
\end{tabular}

Table 2. Observational data of Mars.

Positions of the Sun and Mars in Table 2 are given by the angles from the vernal equinox as shown in Figure 1. The positions of the planets can be described only by the ecliptic longitude if we take the ecliptic coordinate since planetary motions are confined along the ecliptic. It is clear from the small value of the angle inclination of orbit to ecliptic as shown in Table 3. The positive angle of the positions is measured counter clockwise and negative angle is measured clockwise. Since Mars' orbital period of 1.8809 Earth years corresponds to the 678 Earth days, Mars to traverse the orbit by 687 days. The date on the bottom row(E1', E2', E3' and so on) is 687 days later from the date on the top row(E1 E2, E3 and so on) in Table 2. Therefore, Mars is located at same position on the orbit.

\begin{tabular}{|l|c|c|c|c|c|c|}
\hline & Mercury & Venus & Earth & Mars & Jupiter & Saturn \\
\hline Distance (AU) & 0.3871 & 0.7233 & 1 & 1.5237 & 5.2026 & 9.5549 \\
\hline Eccentricity of orbit & 0.2056 & 0.0068 & 0.0167 & 0.0934 & 0.0485 & 0.0555 \\
\hline Inclination of orbit to ecliptic (.) & 7.006 & 3.395 & 0.001 & 1.85 & 1.303 & 2.489 \\
\hline Sidereal period (Y) Year=365.24220ays & 0.2409 & 0.6152 & 1 & 1.8809 & 11.862 & 29.458 \\
\hline Synodic period (day) & 115.9 & 584 & $-\cdots--$ & 779.9 & 398.9 & 378.1 \\
\hline Ecliptic longitude of the perihelion (.) & 77.441 & 131.564 & 102.908 & 336.018 & 14.311 & 93.003 \\
\hline
\end{tabular}

Table 3. Basic data for the planets 


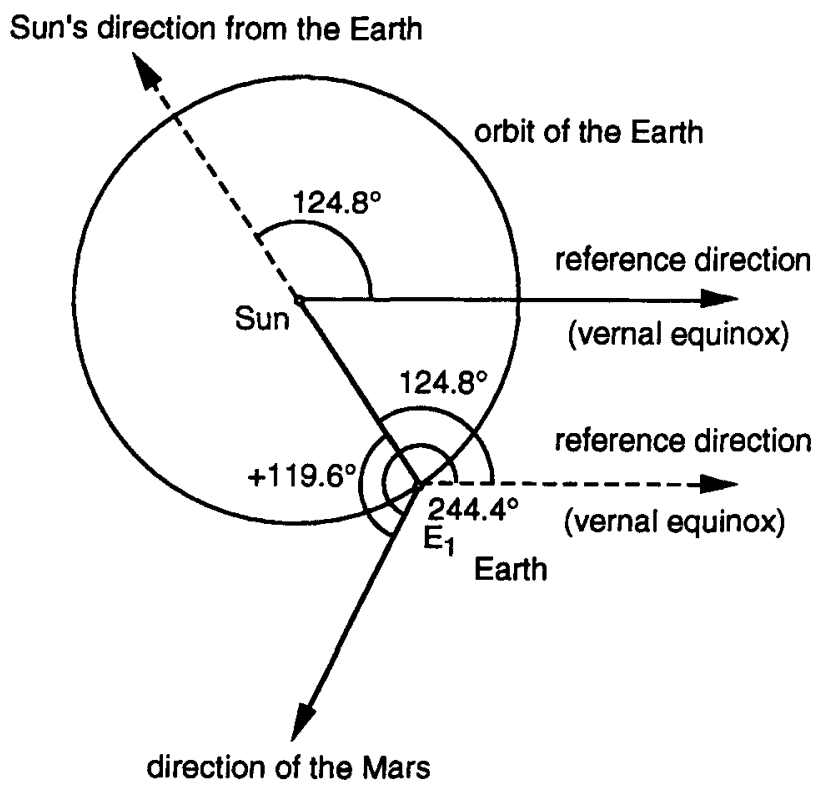

Figure 1. The relationship between the Sun's direction on the celestial sphere and the Earth's position on the orbit.

\subsection{Background}

Students should know certain keywords in order to study planetary motion. The term 'aphelion' and 'perihelion' are the farthest and closest point in an elliptical orbit of a planet, asteroid, or comet from the Sun, respectively. The axis between the aphelion and perihelion is defined as a major axis of the orbit. The half length of the major axis is called as mean distance between a planet and the Sun. The Astronomical Unit (AU), roughly 150 million kilometers, is the mean distance between the Earth and the Sun.

\subsection{Procedure}

(1) Draw a circle with radius of $5 \mathrm{~cm}$ on the center of a sheet of graph paper.

(2) Draw a line from the center of a circle to any direction (e.g., right). Let us assume a circle be the orbit of the Earth, the center the location of the Sun, a line the direction of vernal equinox.

(3) Locate the Earth's position on the orbit using Sun's position in Table 2, which is an angle from the vernal-equinox, as described in Figure 1.

(4) Draw the direction of Mars from the Earth's position using the angle of Mars from vernal equinox in Table 2.

(5) Repeat (3) and (4) for another date within a pair. The intersection of the two lines is the position of the Mars.

(6) Repeat (3) through (5) using other six pairs of observations.

(7) Determine the Martian orbit by connecting seven locations of Mars. Try to draw the Martian orbit as a circle using compass. The center of a circle can be determined by the intersection of the perpendicular bisector of the chords.

(8) Measure and fill the Table 4 using the orbital data drawn in this exercise.

\subsection{Analysis}

(1) Confirm that the center of the Martian orbit is not the Sun. 
(2) Locate the perihelion and aphelion of Mars and measure the counterclockwise angles from the vernal-equinox direction. Determine the mean distance between the Sun and Mars in AU.

Perihelion: degrees from the vernal equinox.

Aphelion: degrees from the vernal equinox.

Mean distance:

$(\mathrm{AU})$

(3) Calculate areal velocity from Table 4 and confirm the Kepler's second law. $\bar{r}$ is the distance between the Sun and the Mars computed as: $\bar{r}=0.5\left(r\left(M_{\mathrm{i}}\right)+r\left(M_{\mathrm{i}+1}\right)\right)$

$A$ and $V$ are the area of the fan-shape sector $\left(S M_{\mathrm{i}} M\right)$ and areal velocity of the Mars respectfully computed as:

$$
A=\pi \bar{r}^{2} \frac{\theta}{360}, V=\frac{A}{t}
$$

where $\theta$ is the center angle of fan-shape area.

(4) Fill Table 5 using your results and calculate the third column in order to confirm the Kepler's third law. $R$ and $P$ are mean distance between the planet and the Sun and orbital period of the planet, respectively.

\begin{tabular}{|l|l|l|l|l|l|l|l|l|}
\hline Mars & Earth & days $(t)$ & $r\left(M_{i}\right)$ & $r\left(M_{i+1}\right)$ & $\bar{r}$ & $\theta$ & $A$ & $V_{\text {area }}$ \\
\hline $\mathrm{M}_{1}-\mathrm{M}_{2}$ & $\mathrm{E}_{1}^{\prime}-\mathrm{E}_{2}$ & 94 & & & & & & \\
\hline $\mathrm{M}_{2}-\mathrm{M}_{3}$ & $\mathrm{E}_{2}^{\prime}-\mathrm{E}_{3}$ & 94 & & & & & & \\
\hline $\mathrm{M}_{3}-\mathrm{M}_{4}$ & $\mathrm{E}_{3}^{\prime}-\mathrm{E}_{4}$ & 94 & & & & & & \\
\hline $\mathrm{M}_{4}-\mathrm{M}_{5}$ & $\mathrm{E}_{4}^{\prime}-\mathrm{E}_{5}$ & 94 & & & & & & \\
\hline $\mathrm{M}_{5}-\mathrm{M}_{6}$ & $\mathrm{E}_{5}^{\prime}-\mathrm{E}_{6}$ & 94 & & & & & & \\
\hline $\mathrm{M}_{6}-\mathrm{M}_{7}$ & $\mathrm{E}_{6}^{\prime}-\mathrm{E}_{7}$ & 94 & & & & & & \\
\hline
\end{tabular}

Table 4. Worksheet for the results

\begin{tabular}{|c|c|c|c|}
\hline Planet & $\mathrm{R}(\mathrm{AU})$ & $\mathrm{P}$ (Year) & $\mathrm{R3} / \mathrm{P2}$ \\
\hline Earth & $1 \mathrm{AU}$ & 1.00 Year & 1 \\
\hline Mars & & 1.88 year & \\
\hline
\end{tabular}

Table 5. Verification of Kepler's Third Law

\subsection{Discussion and Conclusion}

(1) List the assumptions applied in this exercise.

(2) Consider the source of the error of this exercise.

(3) Summarize the Kepler's Law of Planetary Motion. 


\section{Teaching tips}

There are some things to follow to teach Kepler's Law more effectively. First, the radius of the earth's orbit should be two thirds of the radius of the circular protractor. This means that the orbit of Mars corresponds to the size of the circular protractor and hence, it should be easy to identify the center of orbit. The Law of Ellipses states that the Sun's position differs from the center of the orbit. Second, the time intervals between the Mars' positions which are for example, two dates $\mathrm{E} 1$ and E2, should be the same in order to compare the Law of Equal Areas. Mars' areal velocity will be measured by the use of computing a fan-shaped area within Mars' orbital positions about the Sun. An alternative method of measuring the area of a sector is the use of graph paper and count the number of squares in the sector. We may use the simple assumption that all partial squares are count as 0.5 square. Another way uses a accurate balance scale to weigh the paper that fills the sector. We may use the simple assumption that paper's thickness and density are constant. The best method to demonstrate it is by the weights of the fan-shape Mars' trace. Fan-shaped areas will be cut out from the huge cardboard diagram. A visual effect can be created to give an impact to students using a simple balance. Third, the pre-lab activity should include the derivation of the orbital period. The orbital period cannot be measured directly from observation. We can determine only the synodic period which determines the orbital period and hence, is most important period. This exercise is a good problem for algebra. Another pre-lab activity should cover the apparent motion of the planets which include direct and retrograde motions and stationary points since the appropriate theory must explain these planetary motions. Fourth, the post-lab activity should include the analysis of the results. The Law of Period can be confirmed by comparing the results with the Earth. Also, the variation of the planets' orbital velocities should be applied to comets. Halley's comet return every 76 years but the best observation period lasts only a year. That is because of the Kepler's second law of planetary motion. The Earth's orbit was assumed to be circlar in this activity and hence, contradict the results that identifies it as elliptical. However, the eccentricity of the earth is only 0.0167 corresponding the $0.84 \mathrm{~mm}$ in the diagram as compared with Mars' 0.0934 corresponding the $7.12 \mathrm{~mm}$. Therefore, we can neglect the eccentricity of the earth in this activity. There are revelations in this activity. Students will learn the history of science and the process of a geocentric system versus a heliocentric system. That is not an easy process because the idea is counter to our common sense. Scientific belief is often a hypothesis. The hypothesis should be the most-reliable idea at that time. However, it might be replaced by a newly-developed hypothesis. That is the nature of scientific belief.

\section{Summary}

Instructors should bear in mind that this is a kind of 'integrated curriculum'. Students need to utilize and apply their knowledge of mathematics, physics, earth science, and history of science. Data was computed by Stellar Navigator (a commercial software in Japan). Moreover, diagrams presented here were drawn by Mac Draw Pro on a Macintosh computer. Therefore, this is not only teaching astronomy but also scientific method. It requires simple materials (ruler, circular protractor, compass, and graph paper) and hence can be done at any school all over the world. The most recent observational data will be supplied on the Internet from Keio Senior High School(www.ifet.or.jp/ keio). 


\section{REFERENCES}

Churttrand, M. R. \& Tirion, W., 1991, The Audubon Society Field Guide to the Night Sky, Alfred Knopf, Inc., New York.

Morrison, D \& Owen, T., 1988, The Planetary System, Addison-Wesley, New York.

Many Japanese Standard Textbooks

\section{Appendix}

\section{Grade _ Student ID\# __ Name}

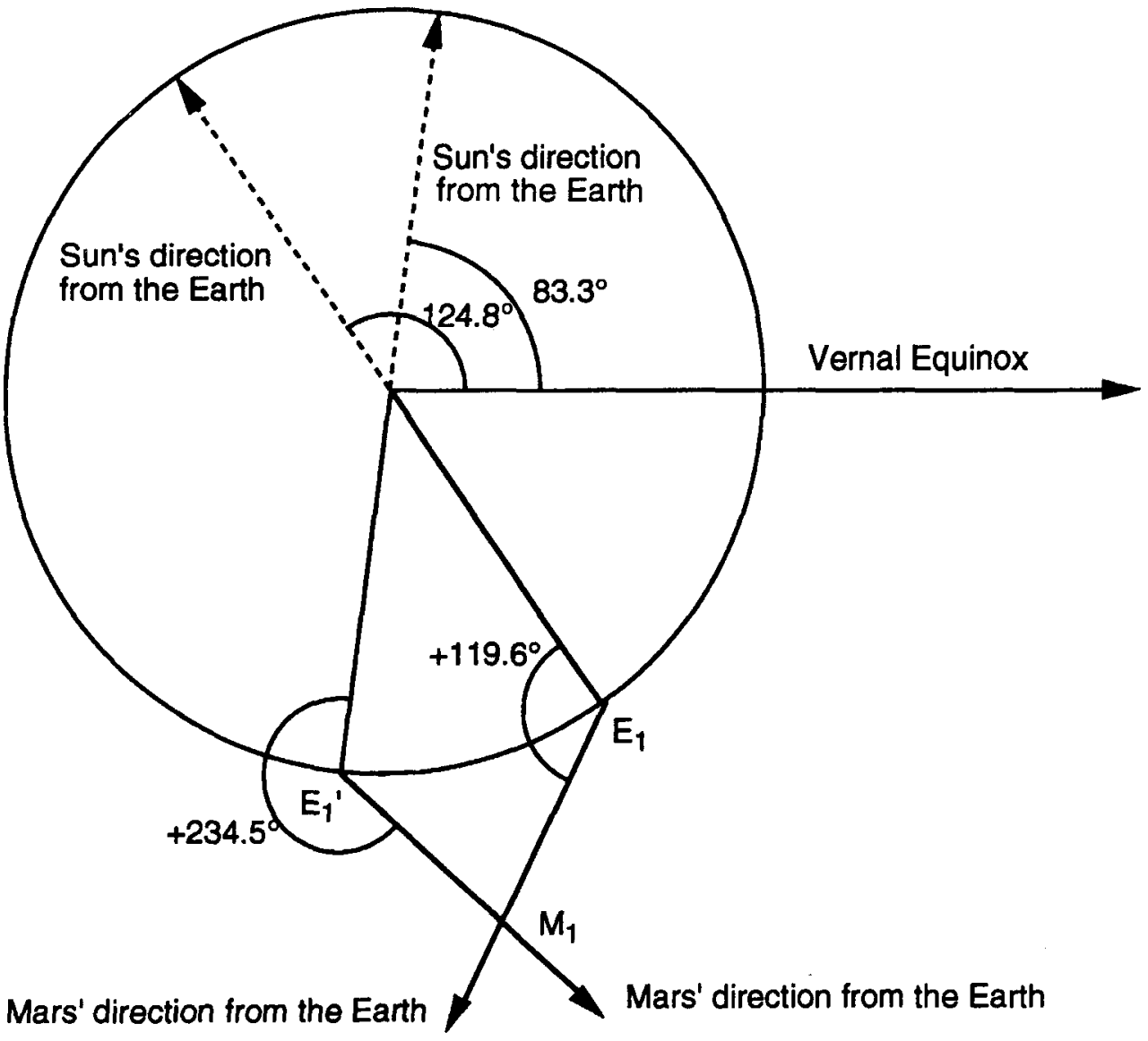

FIGURE 2. Worksheet for classroom use. 